\begin{tabular}{|c|l|}
\hline Title & A class of Hilbert space representations of the quantum plane and the quantum algebra U q(s/2) \\
\hline Author(s) & A rai, A sao \\
\hline Citation & $\begin{array}{l}\text { Reports on Mathematical Physics, 61(2), 171-180 } \\
\text { https://doi.org/10.1016/S0034 4877(08)00013X }\end{array}$ \\
\hline Issue Date & 2008-04 \\
\hline Doc URL & http://hdl.handle.net/2115/38249 \\
\hline Type & article (author version) \\
\hline File Information & arai-rev1.pdf \\
\hline
\end{tabular}

Instructions for use 


\title{
A Class of Hilbert Space Representations of the Quantum Plane and the Quantum Algebra $U_{q}\left(\mathrm{sl}_{2}\right)$
}

\author{
Asao Arai * \\ Department of Mathematics, Hokkaido University \\ Sapporo 060-0810, Japan \\ e-mail: arai@math.sci.hokudai.ac.jp
}

\begin{abstract}
A class of representations on the Hilbert space $L^{2}\left(\mathbb{R}^{d}\right)(d \geq 2)$ of the quantum plane $\mathbb{C}_{q}^{2}$ and the quantum algebra $U_{q}\left(\mathrm{sl}_{2}\right)$ is presented. The boundedness and the unboundedness of the representations are discussed. A physically interesting example of the representations is shown to appear in a two-dimensional quantum system with a magnetic field concentrated on an infinite lattice.
\end{abstract}

Keywords: quantum plane, quantum algebra, singular magnetic field.

\section{Introduction}

In the previous paper [2] (cf. also [1]), the author considered a quantum system in the plane $\mathbb{R}^{2}$ which is under the influence of a perpendicular magnetic field concentrated on an infinite discrete set $\mathbf{D}$ of $\mathbb{R}^{2}$ and showed that, in this quantum system, there appear, in a natural way, representations of the canonical commutation relations (CCR) with two degrees of freedom. Interestingly enough, these representations are not necessarily unitarily equivalent to the Schrödinger representation of the CCR with two degrees of freedom and each inequivalent representation physically corresponds to the occurrence of the Aharonov-Bohm effect in the context of the quantum system under consideration. Moreover, in connection with the inequivalent representations with $\mathbf{D}$ being an infinite lattice, bounded operator representations of the quantum plane $\mathbb{C}_{q}^{2}$ and the quantum algebra $U_{q}\left(\mathrm{sl}_{2}\right)$ with $|q|=1(q \in \mathbb{C})$ were constructed as well as their reductions to lattice quantum systems.

*Supported by the Grant-In-Aid No.17340032 for Scientific Research from the JSPS. 
From a mathematical point of view, it would be natural to ask if there is any general structure behind the representations of $\mathbb{C}_{q}^{2}(|q|=1)$ constructed in [2]. This is one of the motivations for the present work. In this paper, we show that the answer is affirmative, presenting a general theory of representations of $\mathbb{C}_{q}^{2}$ on the Hilbert space $L^{2}\left(\mathbb{R}^{d}\right)(d \geq 2)$ with $|q|$ not necessarily equal to one. This general theory, of course, includes, as examples, the previously obtained representations of $\mathbb{C}_{q}^{2}$ mentioned above. A new feature of the representations of $\mathbb{C}_{q}^{2}$ given in this paper is in that they may be unbounded.

As for work related to the present one, we mention only Schmüdgen's papers $[6,7,8]$, which may have close connections in theoretical structures and mathematical methods. In [6] (resp. [7]), *-representations of the real quantum plane $\mathbb{R}_{q}^{2}$ (resp. $\mathbb{R}_{q}^{2}$, the real quantum hyperboloid $X_{q, \gamma}$ and $S L_{q}(2, \mathbb{R})$ ) with $|q|=1$ are discussed, while those of $\mathcal{U}_{q}\left(\operatorname{sl}_{2}(\mathbb{R})\right)$ with $|q|=1$ are investigated in [8]. We remark that, in the present paper, we do not consider $*$-structures of the representations constructed.

The present paper is organized as follows. Section 2 is a preliminary, where we define a class of closed linear operators on $L^{2}\left(\mathbb{R}^{d}\right)$ constructed from the Schrödinger representation of the CCR with $d$ degrees of freedom. In Section 3 we present a general scheme for the construction of a class of representations of $\mathbb{C}_{q}^{2}$ on $L^{2}\left(\mathbb{R}^{d}\right)$. Here we see that, to obtain an unbounded operator representation, the constituent operators of the representation are required to have a common invariant domain. A possible form of such an invariant domain is given in Section 4 in the case $d=2$. In the paper [1], we showed a scheme to obtain representations of $U_{q}\left(\mathrm{sl}_{2}\right)$ from a representation of $\mathbb{C}_{q}^{2}$. Using this scheme and the results in Section 3, we present in Section 5 a class of representations of $U_{q}\left(\mathrm{sl}_{2}\right)$ on $L^{2}\left(\mathbb{R}^{d}\right)$. In the last section, we consider a two dimensional quantum system with a singular gauge potential determined by a meromorphic function of which poles are on an infinite lattice and show that representations of $\mathbb{C}_{q}^{2}$ (resp. $U_{q}\left(\mathrm{sl}_{2}\right)$ ) of the type discussed in Section 3 (resp. Section 5 ) are realized in this system.

\section{Preliminary}

For each vector $\mathbf{a} \in \mathbb{R}^{d}(d \geq 2)$, we define a unitary operator $V(\mathbf{a})$ on the Hilbert space $L^{2}\left(\mathbb{R}^{d}\right)$ by

$$
(V(\mathbf{a}) \psi)(\mathbf{x}):=\psi(\mathbf{x}+\mathbf{a}), \quad \psi \in L^{2}\left(\mathbb{R}^{d}\right), \text { a.e.(almost everywhere) } \mathbf{x} \in \mathbb{R}^{d} .
$$

Let $G$ be a Borel measurable function on $\mathbb{R}^{d}$ a.e. finite. We denote the multiplication operator by the function $G$ on $L^{2}\left(\mathbb{R}^{d}\right)$ by the same symbol $G$. The following lemma is well-known (or easily proved):

Lemma 1 Let $G_{\mathbf{a}}(\mathbf{x}):=G(\mathbf{x}+\mathbf{a})$, a.e. $\mathbf{x} \in \mathbb{R}^{d}$. Then, for all $\mathbf{a} \in \mathbb{R}^{d}$, the operator equality $V(\mathbf{a}) G V(\mathbf{a})^{*}=G_{\mathbf{a}}$ holds, where $V(\mathbf{a})^{*}$ denotes the adjoint of $V(\mathbf{a})$. 
Let

$$
T(\mathbf{a}):=G V(\mathbf{a}) .
$$

Since the function $G$ is a.e. finite, the multiplication operator $G$ is a densely defined closed linear operator on $L^{2}\left(\mathbb{R}^{d}\right)$. It follows that the same holds for $T(\mathbf{a})$. But $T(\mathbf{a})$ may be unbounded. Note that the operator equality

$$
G=T(\mathbf{a}) V(\mathbf{a})^{-1}
$$

holds. Some basic properties of the operator $T(\mathbf{a})$ are summarized in the next proposition:

Proposition 1 Let $T(\mathbf{a})$ be as above. Then:

(i) The operator $T(\mathbf{a})$ is bounded if and only if the function $G$ is essentially bounded on $\mathbb{R}^{d}$. In that case, $D(T(\mathbf{a}))=D(G)=L^{2}\left(\mathbb{R}^{d}\right)$.

(ii) The operator $T(\mathbf{a})$ is unitary if and only if $|G(\mathbf{x})|=1$, a.e. $\mathbf{x} \in \mathbb{R}^{d}$.

(iii) Let

$$
N_{G}:=\left\{\mathbf{x} \in \mathbb{R}^{d} \mid G(\mathbf{x})=0\right\} .
$$

Then $T(\mathbf{a})$ is injective if and only if the Lebesgue measure $\left|N_{G}\right|$ of $N_{G}$ is equal to zero. In that case, $G$ is injective with $T(\mathbf{a})^{-1}=V(-\mathbf{a}) G^{-1}$ on $D\left(G^{-1}\right)$.

Proof. (i) Let $T(\mathbf{a})$ be bounded. Then it follows from the closedness of $T(\mathbf{a})$ and the denseness of $D(T(\mathbf{a}))$ that $D(T(\mathbf{a}))=L^{2}\left(\mathbb{R}^{d}\right)$. Hence, by (3), $G$ is bounded with $D(G)=L^{2}\left(\mathbb{R}^{d}\right)$, implying that the function $G$ is essentially bounded on $\mathbb{R}^{d}$. Proving the converse statement is easy.

(ii) If $T(\mathbf{a})$ is unitary, then, by (3), $G$ is unitary. This implies that $|G(\mathbf{x})|=$ 1 , a.e.x $\in \mathbb{R}^{d}$. The converse statement can be easily proved.

(iii) Suppose that $T(\mathbf{a})$ is injective. Then, by (3), $G$ is injective. On the other hand, it is easy to see that $\operatorname{ker} G=\left\{\psi \in L^{2}\left(\mathbb{R}^{d}\right) \mid\left\{\mathbf{x} \in K_{\psi}^{c} \mid \psi(\mathbf{x}) \neq 0\right\} \subset\right.$ $\left.N_{G}\right\} \cdots(*)$, where $K_{\psi}$ is a null set of $\mathbb{R}^{d}$ depending on $\psi$. Hence $\left|N_{G}\right|$ must be zero. Conversely, suppose that $\left|N_{G}\right|=0$. Then, by $(*), \operatorname{ker} G=\{0\}$. Hence $G$ is injective, which, together with the injectivity of $V(\mathbf{a})$, implies that $T(\mathbf{a})$ is injective.

For each vector $\mathbf{b} \in \mathbb{R}^{d}$, we define a unitary operator

$$
U(\mathbf{b}):=e^{i \mathbf{b} \cdot \mathbf{x}}
$$

(the multiplication operator by the function $\left.: \mathbf{x} \mapsto e^{i \mathbf{b} \cdot \mathbf{x}}\right)$ on $L^{2}\left(\mathbb{R}^{d}\right)$. It is easy to see that

$$
V(\mathbf{a}) U(\mathbf{b})=e^{i \mathbf{a} \cdot \mathbf{b}} U(\mathbf{b}) V(\mathbf{a}), \quad \mathbf{a}, \mathbf{b} \in \mathbb{R}^{d},
$$

the $d$-dimensional form of the Weyl relation.

Proposition 2 For all $\mathbf{a}, \mathbf{b} \in \mathbb{R}^{d}, U(\mathbf{b}) D(T(\mathbf{a}))=D(T(\mathbf{a}))$ and

$$
U(\mathbf{b}) T(\mathbf{a})=e^{-i \mathbf{a} \cdot \mathbf{b}} T(\mathbf{a}) U(\mathbf{b}) .
$$


Proof. Direct computation using (6) and the commutativity of $G$ with $U(\mathbf{b})$.

Proposition 3 For all $\mathbf{a} \in \mathbb{R}^{d}, T(\mathbf{a})^{*}=\left(G^{*}\right)_{-\mathbf{a}} V(-\mathbf{a})$ and $D(G) \subset D\left(T(\mathbf{a})^{*}\right)$.

Proof. By Lemma 1, we have $T(\mathbf{a})=V(\mathbf{a}) G_{-\mathbf{a}}$. Since $V(\mathbf{a})$ is bounded, it follows that $T(\mathbf{a})^{*}=G_{-\mathbf{a}}^{*} V(\mathbf{a})^{*}=\left(G^{*}\right)_{-\mathbf{a}} V(-\mathbf{a})$. Let $\psi \in D(G)$. Then

$$
\int_{\mathbb{R}^{d}}\left|\left(G^{*}\right)_{-\mathbf{a}}(\mathbf{x})\right|^{2}|(V(-\mathbf{a}) \psi)(\mathbf{x})|^{2} d \mathbf{x}=\int_{\mathbb{R}^{d}}|G(\mathbf{x})|^{2}|\psi(\mathbf{x})|^{2} d \mathbf{x}<\infty .
$$

Hence $\psi \in D\left(T(\mathbf{a})^{*}\right)$.

\section{A Class of Hilbert Space Representations of the Quantum Plane}

Let $\mathbf{w}_{1}$ and $\mathbf{w}_{2}$ be linearly independent vectors in $\mathbb{R}^{d}$. Suppose that there exist non-zero Borel measurable functions $F_{j}: \mathbb{R}^{d} \rightarrow \mathbb{C}(j=1,2)$ a.e. finite and a constant $q \in \mathbb{C} \backslash\{0\}$ satisfying

$$
F_{1}(\mathbf{x}) F_{2}\left(\mathbf{x}+\mathbf{w}_{1}\right)=q F_{1}\left(\mathbf{x}+\mathbf{w}_{2}\right) F_{2}(\mathbf{x}), \quad \text { a.e. } \mathbf{x} \in \mathbb{R}^{d} .
$$

A simple example satisfying the functional equation (7) is given as follows:

Example 1 Let $\mathbf{u}, \mathbf{v} \in \mathbb{R}^{d}, \alpha, \beta \in \mathbb{C}$ and $q:=e^{\beta \mathbf{w}_{1} \cdot \mathbf{v}-\alpha \mathbf{w}_{2} \cdot \mathbf{u}}$. Let

$$
F_{1}(\mathbf{x}):=e^{\alpha \mathbf{x} \cdot \mathbf{u}}, \quad F_{2}(\mathbf{x}):=e^{\beta \mathbf{x} \cdot \mathbf{v}} .
$$

Then it is easily checked that these $F_{1}$ and $F_{2}$ satisfy (7).

We define

$$
T_{j}:=F_{j} V\left(\mathbf{w}_{j}\right), \quad j=1,2 .
$$

Theorem 1 For all $\psi \in D\left(T_{1}^{*}\right) \cap D\left(T_{2}^{*}\right)$ and $\phi \in D\left(T_{1}\right) \cap D\left(T_{2}\right)$,

$$
\left\langle T_{1}^{*} \psi, T_{2} \phi\right\rangle=q\left\langle T_{2}^{*} \psi, T_{1} \phi\right\rangle
$$

where $\langle\cdot, \cdot\rangle$ denotes the inner product of $L^{2}\left(\mathbb{R}^{d}\right)$.

Proof. We have $\left\langle T_{1}^{*} \psi, T_{2} \phi\right\rangle=\int_{\mathbb{R}^{d}} F_{1}\left(\mathbf{x}-\mathbf{w}_{1}\right) F_{2}(\mathbf{x}) \psi\left(\mathbf{x}-\mathbf{w}_{1}\right)^{*} \phi\left(\mathbf{x}+\mathbf{w}_{2}\right) d \mathbf{x}$ and

$$
\left\langle T_{2}^{*} \psi, T_{1} \phi\right\rangle=\int_{\mathbb{R}^{d}} F_{2}\left(\mathbf{x}-\mathbf{w}_{2}\right) F_{1}(\mathbf{x}) \psi\left(\mathbf{x}-\mathbf{w}_{2}\right)^{*} \phi\left(\mathbf{x}+\mathbf{w}_{1}\right) d \mathbf{x} .
$$

By change of variables and condition (7), one sees that (9) holds.

Theorem 1 and Proposition 1-(i) imply the following result: 
Corollary 1 Suppose that $F_{1}$ and $F_{2}$ are essentially bounded on $\mathbb{R}^{d}$. Then $T_{1}$ and $T_{2}$ are bounded with $D\left(T_{j}\right)=L^{2}\left(\mathbb{R}^{d}\right)(j=1,2)$ and $T_{1} T_{2}=q T_{2} T_{1}$.

Thus, in the case where $F_{1}$ and $F_{2}$ are essentially bounded, the set $\left\{T_{1}, T_{2}\right\}$ of operators yields a bounded operator representation of the quantum plane $\mathbb{C}_{q}^{2}$ with $q \neq 0, \pm 1([4,5])$.

In the case where at least one of $F_{1}$ and $F_{2}$ is not essentially bounded on $\mathbb{R}^{d}$, however, we need some condition for $\left\{T_{1}, T_{2}\right\}$ to give a representation of $\mathbb{C}_{q}^{2}$ :

Corollary 2 Suppose that there exists a dense subspace $D$ in $L^{2}\left(\mathbb{R}^{d}\right)$ such that, for $j=1,2, V\left(\mathbf{w}_{j}\right) D \subset D$ and $F_{j} D \subset D$. Then $T_{j} D \subset D(j=1,2)$ and $T_{1} T_{2}=q T_{2} T_{1}$ on $D$.

Proof. The property $T_{j} D \subset D$ follows from the present assumption and the definition of $T_{j}$. By Proposition $3, D \subset D\left(T_{1}^{*}\right) \cap D\left(T_{2}^{*}\right)$. Hence, by Theorem 1, we have that, for all $\psi, \phi \in D,\left\langle\psi, T_{1} T_{2} \phi\right\rangle=\left\langle\psi, q T_{2} T_{1} \phi\right\rangle$. Since $D$ is dense, we obtain the desired result.

Suppose that the assumption of Corollary 2 holds. Then we can define the algebra generated by $T_{1} \mid D$ (the restriction of $T_{1}$ to $D$ ) and $T_{2} \mid D$. We denote it $\mathcal{O}_{\mathbf{w}_{1}, \mathbf{w}_{2}}(D)$. We define

$$
\mathcal{O}_{\mathbf{w}_{1}, \mathbf{w}_{2}}(D)^{\prime}:=\left\{B \in \mathfrak{B}\left(L^{2}\left(\mathbb{R}^{d}\right)\right) \mid\left\langle B^{*} \phi, T_{j} \psi\right\rangle=\left\langle T_{j}^{*} \phi, B \psi\right\rangle, \phi, \psi \in D, j=1,2\right\},
$$

the weak commutant of $\mathcal{O}_{\mathbf{w}_{1}, \mathbf{w}_{2}}(D)$, where $\mathfrak{B}\left(L^{2}\left(\mathbb{R}^{d}\right)\right)$ denotes the Banach space of all bounded linear operators $B$ on $L^{2}\left(\mathbb{R}^{d}\right)$ with $D(B)=L^{2}\left(\mathbb{R}^{d}\right)$.

Proposition 4 Let $\mathbf{a} \in \mathbb{R}^{d}$ be such that $\mathbf{a} \cdot \mathbf{w}_{j} \in 2 \pi \mathbb{Z}, j=1,2$ and $U(\mathbf{a}) D \subset D$. Then $U(\mathbf{a}) \in \mathcal{O}_{\mathbf{w}_{1}, \mathbf{w}_{2}}(D)^{\prime}$.

Proof. Applying Proposition 2, we have $U(\mathbf{a}) T_{j}=T_{j} U(\mathbf{a})$, which implies the desired result.

\section{Invariant Domains in the Case $d=2$}

In this section, we consider the case $d=2$ and suppose that $\mathbf{w}_{1} \times \mathbf{w}_{2}>0$. In this case, a possible invariant domain for $T_{1}$ and $T_{2}$ can be constructed as follows. We first introduce a subset $\mathbb{L}_{j}$ of $\mathbb{R}^{d}(j=1,2)$ by

$$
\mathbb{L}_{1}:=\cup_{n \in \mathbb{Z}}\left\{t \mathbf{w}_{1}+n \mathbf{w}_{2} \mid t \in \mathbb{R}\right\}, \quad \mathbb{L}_{2}:=\cup_{m \in \mathbb{Z}}\left\{m \mathbf{w}_{1}+t \mathbf{w}_{2} \mid t \in \mathbb{R}\right\} .
$$

Note that

$$
\mathbb{L}_{1} \cap \mathbb{L}_{2}=\mathbb{L}_{\mathbf{w}_{1}, \mathbf{w}_{2}}:=\left\{m \mathbf{w}_{1}+n \mathbf{w}_{2} \mid m, n \in \mathbb{Z}\right\},
$$

a two-dimensional lattice. The set

$$
\Omega:=\mathbb{L}_{1}^{c} \cap \mathbb{L}_{2}^{c}=\left(\mathbb{L}_{1} \cup \mathbb{L}_{2}\right)^{c}
$$


is an open set in $\mathbb{R}^{d}$. It is easy to see that

$$
\Omega \pm \mathbf{w}_{j}=\Omega, \quad j=1,2 .
$$

Hence $\Omega$ has the translation symmetry with vectors $\pm \mathbf{w}_{j}(j=1,2)$. We denote by $C_{0}^{\infty}(\Omega)$ the set of infinitely differentiable functions on $\mathbb{R}^{d}$ with compact support in $\Omega$.

Proposition 5 Suppose that each $F_{j}(j=1,2)$ is infinitely differentiable on $\Omega$. Then $T_{j} C_{0}^{\infty}(\Omega) \subset C_{0}^{\infty}(\Omega)$ and $T_{1} T_{2}=q T_{2} T_{1}$ on $C_{0}^{\infty}(\Omega)$.

Proof. We have $F_{j} C_{0}^{\infty}(\Omega) \subset C_{0}^{\infty}(\Omega)$. By $(14), V\left(\mathbf{w}_{j}\right) C_{0}^{\infty}(\Omega) \subset C_{0}^{\infty}(\Omega)$. Thus the desired result follows.

\section{A Class of Hilbert Space Representations of $U_{q}\left(\mathrm{sl}_{2}\right)$}

For a complex number $q \in \mathbb{C} \backslash\{0,-1,1\}$, the quantum algebra $U_{q}\left(\mathrm{sl}_{2}\right)$ is defined to be the complex associative algebra with unit 1 generated by four elements $E, F, K, K^{-1}$ subject to the following relations $[4,5]$ :

$$
\begin{aligned}
& K K^{-1}=K^{-1} K=1, \quad K E K^{-1}=q^{2} E, \quad K F K^{-1}=q^{-2} F, \\
& {[E, F]=\frac{K-K^{-1}}{q-q^{-1}},}
\end{aligned}
$$

where $[X, Y]:=X Y-Y X$. As is shown in Lemma 4.1 and Lemma 5.1 in [1] (cf. also [9]), there is a general scheme to construct a representation of $U_{q}\left(\mathrm{sl}_{2}\right)$ from a representation of the quantum plane $\mathbb{C}_{q}^{2}$.

Let $T_{1}$ and $T_{2}$ be as in Section 3 .

Theorem 2 Assume that $\left|N_{F_{j}}\right|=0, j=1,2$. Suppose that there exists a dense subspace $D$ in $L^{2}\left(\mathbb{R}^{d}\right)$ such that, for $j=1,2, V\left(\mathbf{w}_{j}\right) D \subset D$ and $F_{j} D \subset$ $D, F_{j}^{-1} D \subset D$. Let $a, b, a^{\prime}, b^{\prime} \in \mathbb{C}$ be constants satisfying

$$
a b q=\frac{a^{\prime} b^{\prime}}{q}=-\frac{1}{\left(q-q^{-1}\right)^{2}} .
$$

and

$$
\begin{aligned}
& E:=T_{2}\left(a T_{2}+a^{\prime} T_{2}^{-1}\right) T_{1}^{-1}, \quad F:=T_{1}\left(b T_{2}+b^{\prime} T_{2}^{-1}\right) T_{2}^{-1}, \\
& K:=T_{2}^{2}, \quad K^{-1}:=\left(T_{2}^{-1}\right)^{2} .
\end{aligned}
$$

Then the set $\left\{E, F, K, K^{-1}\right\}$ generates a representation of $U_{q}\left(\mathrm{sl}_{2}\right)$ on the vector space $D$.

Proof. Apply Lemma 4.1 in [1] to the representation $\left\{T_{1}, T_{2}\right\}$ of $\mathbb{C}_{q}^{2}$.

We denote by $\Pi_{a, b ; a^{\prime}, b^{\prime}}$ the representation of $U_{q}\left(\mathrm{sl}_{2}\right)$ given in Theorem 2 . 
Proposition 6 There is no finite dimensional subspace $M$ of $D$ which reduces the representation $\Pi_{a, b ; a^{\prime}, b^{\prime}}$ and in which the highest weight of $\Pi_{a, b ; a^{\prime}, b^{\prime}} \mid M$ is not equal to $-a^{\prime} / a q^{2}$.

Proof. Suppose that there is a finite dimensional subspace $M$ of $D$ which reduces the representation $\Pi_{a, b ; a^{\prime}, b^{\prime}}$. By a general fact on finite dimensional representations of $U_{q}\left(\mathrm{sl}_{2}\right)$ ([4, Proposition VI.3.3]), The representation $\Pi_{a, b ; a^{\prime}, b^{\prime}} \mid M$ has a highest weight vector $\psi \in M \backslash\{0\}: E \psi=0$ and $K \psi=\lambda \psi$ with highest weight $\lambda \in \mathbb{C} \backslash\{0\}$. Then

$$
\begin{aligned}
& T_{2}\left(a T_{2}+a^{\prime} T_{2}^{-1}\right) T_{1}^{-1} \psi=0, \\
& F_{2}(\mathbf{x}) F_{2}\left(\mathbf{x}+\mathbf{w}_{2}\right) \psi\left(\mathbf{x}+2 \mathbf{w}_{2}\right)=\lambda \psi(\mathbf{x}), \quad \text { a.e. } \mathbf{x} \in \mathbb{R}^{d} .
\end{aligned}
$$

By (20) and the easily derived relation $T_{2} T_{1}^{-1}=q T_{1}^{-1} T_{2}$, we obtain $\left(a q^{2} \lambda+\right.$ $\left.a^{\prime}\right) T_{1}^{-1} \psi=0$. Hence $a q^{2} \lambda+a^{\prime} \neq 0$. Therefore $\lambda=-a^{\prime} / a q^{2}$. This implies the desired assertion.

Theorem 3 Suppose that the following (i) or (ii) hold:

(i) For a constant $c>0,\left|F_{2}(\mathbf{x})\right|=c$, a.e. $\mathbf{x} \in \mathbb{R}^{d}$.

(ii) $\left|F_{2}(\mathbf{x})\right|^{2} \geq\left|a^{\prime}\right| /|a||q|^{2}$, a.e. $\mathbf{x} \in \mathbb{R}^{d}$.

Then there is no finite dimentional subspace $M$ of $D$ which reduces the representation $\Pi_{a, b ; a^{\prime}, b^{\prime}}$.

Proof. By a general fact on finite dimensional representations of $U_{q}\left(\mathrm{sl}_{2}\right)$ ([4, Proposition VI.3.3]), we need only to show that the representation $\Pi_{a, b ; a^{\prime}, b^{\prime}}$ has no highest weight vector. Let $\psi$ be a vector in $D$ such that $E \psi=0$ and $K \psi=\lambda \psi$ with $\lambda \in \mathbb{C} \backslash\{0\}$. Then (20) and (21) hold. By Proposition 6, we need only to consider the case $\lambda=-a^{\prime} / a q^{2} \neq 0$. Then, by (21), we have $\left|F_{2}(\mathbf{x})\right|\left|F_{2}\left(\mathbf{x}+\mathbf{w}_{2}\right)\right|\left|\psi\left(\mathbf{x}+2 \mathbf{w}_{2}\right)\right|=\left.\left|a^{\prime}\right||\psi(\mathbf{x}) /| a|| q\right|^{2}$.

Let condition (i) be satisfied. Then we have $k\left|\psi\left(\mathbf{x}+2 \mathbf{w}_{2}\right)\right|=|\psi(\mathbf{x})|$ with $k=|a||q|^{2} c^{2} /\left|a^{\prime}\right|$. Since $\psi$ is in $L^{2}\left(\mathbb{R}^{d}\right)$, it follows that $k=1$. Then $\mid \psi(\mathbf{x}+$ $\left.2 \mathbf{w}_{2}\right)|=| \psi(\mathbf{x}) \mid$. But this implies $\int_{\mathbb{R}^{d}}|\psi(\mathbf{x})|^{2} d x=\infty$ if $\psi \neq 0$. Thus $\psi=0$.

We next consider the case where condition (ii) holds. In this case we have $\left|\psi\left(\mathbf{x}+2 \mathbf{w}_{2}\right)\right| \leq \mid \psi(\mathbf{x})$. This inequality and condition $\psi \in L^{2}\left(\mathbb{R}^{d}\right)$ imply that $\psi=0$.

The element $C$ defined by

$$
C:=\frac{q K-2+q^{-1} K^{-1}}{\left(q-q^{-1}\right)^{2}}+F E
$$

is called the Casimir element of $U_{q}\left(\mathrm{sl}_{2}\right)$ and commutes with $E, F, K$ and $K^{-1}$.

In the representation $\Pi_{a, b ; a^{\prime}, b^{\prime}}$, we have

$$
\Pi_{a, b ; a^{\prime}, b^{\prime}}(C)=a^{\prime} b+a b^{\prime}-\frac{2}{\left(q-q^{-1}\right)^{2}},
$$

which is a scalar. 
Theorem 4 Let $\Pi_{a, b ; a^{\prime}, b^{\prime}}$ and $\Pi_{c, d ; c^{\prime}, d^{\prime}}$ be two representations of $U_{q}\left(\mathrm{sl}_{2}\right)$ of the form given in Theorem 2. Suppose that $a^{\prime} b+a b^{\prime} \neq c^{\prime} d+c d^{\prime}$. Then $\prod_{a, b ; a^{\prime}, b^{\prime}}$ is not unitarily equivalent to $\Pi_{c, d ; c^{\prime}, d^{\prime}}$.

Proof. If $\Pi_{a, b ; a^{\prime}, b^{\prime}}$ is unitarily equivalent to $\Pi_{c, d ; c^{\prime}, d^{\prime}}$ with a unitary operator $U$, then $U \Pi_{a, b ; a^{\prime}, b^{\prime}}(C) U^{-1}=\Pi_{c, d ; c^{\prime}, d^{\prime}}(C)$. Hence, by (23), we have $a^{\prime} b+a b^{\prime}=$ $c^{\prime} d+c d^{\prime}$. But this is a contradiction.

Proposition 7 Let $F_{1}$ and $F_{2}$ be essentially bounded and $\mathfrak{A}_{q}$ be the *-algebra generated by E, F, K, $K^{-1}$ in Theorem 2. Then $\mathfrak{A}_{q}$ is not irreducible.

Proof. Let $W$ be the two-dimensional subspace generated by $\mathbf{w}_{1}$ and $\mathbf{w}_{2}$ and $\left\{\mathbf{f}_{1}, \mathbf{f}_{2}\right\}$ be an orthonormal basis of $W$. We expand $\mathbf{w}_{j}(j=1,2)$ as $\mathbf{w}_{j}=$ $w_{j 1} \mathbf{f}_{1}+w_{j 2} \mathbf{f}_{2}\left(w_{j 1}, w_{j 2} \in \mathbb{C}\right)$ and define a matrix

$$
T:=\left(\begin{array}{ll}
w_{11} & w_{12} \\
w_{21} & w_{22}
\end{array}\right)
$$

Then $T$ is regular. For each $\mathbf{n}=\left(n_{1}, n_{2}\right) \in \mathbb{Z} \times \mathbb{Z}$, we define $\left(a_{\mathbf{n} 1}, a_{\mathbf{n} 2}\right) \in \mathbb{C}^{2}$ by $\left(a_{\mathbf{n} 1}, a_{\mathbf{n} 2}\right):=2 \pi T^{-1} \mathbf{n}$. Then the vector $\mathbf{a}_{\mathbf{n}}:=a_{\mathbf{n} 1} \mathbf{f}_{1}+a_{\mathbf{n} 2} \mathbf{f}_{2}$ satisfies $\mathbf{a}_{\mathbf{n}} \cdot \mathbf{w}_{j}=$ $2 \pi n_{j} \in 2 \pi \mathbb{Z}$. Hence $U\left( \pm \mathbf{a}_{\mathbf{n}}\right)$ commute with $E, F, K$ and $K^{-1}$, and hence all those of $\mathfrak{A}_{q}$ (note that $U\left(\mathbf{a}_{\mathbf{n}}\right)^{*}=U\left(-\mathbf{a}_{\mathbf{n}}\right)$ ). Therefore the commutant of $\mathfrak{A}_{q}$ is not the set of scalar operators. Thus $\mathfrak{A}_{q}$ is not irreducible.

\section{Representations of $\mathbb{C}_{q}^{2}$ and $U_{q}\left(\mathrm{sl}_{2}\right)$ in a quantum system with a singular gauge potential}

In this section we consider a two-dimensional quantum system with a perpendicular magnetic field concentrated on the infinite lattice $\mathbb{L}_{\mathbf{w}_{1}, \mathbf{w}_{2}}$ defined by (12).

For a vector $\mathbf{a}=\left(a_{1}, a_{2}\right)$, we denote its corresponding complex number by $a=a_{1}+i a_{2}$.

Let $f$ be a meromorphic function on $\mathbb{C} \backslash\left\{n w_{1}+m w_{2} \mid m, n \in \mathbb{Z}\right\}$ with possible poles on the points $n w_{1}+m w_{2}(m, n \in \mathbb{Z})\left(w_{j}=w_{j 1}+i w_{j 2}\right)$. Then one can define a gauge potential $\mathbf{A}$ on

$$
\mathbb{M}:=\mathbb{R}^{2} \backslash \mathbb{L}_{\mathbf{w}_{1}, \mathbf{w}_{2}}
$$

by

$$
\mathbf{A}(\mathbf{x})=\left(A_{1}(\mathbf{x}), A_{2}(\mathbf{x})\right)
$$

with

$$
A_{1}(\mathbf{x}):=\Im f\left(x_{1}+i x_{2}\right), \quad A_{2}(\mathbf{x}):=\Re f\left(x_{1}+i x_{2}\right), \quad \mathbf{x}=\left(x_{1}, x_{2}\right) \in \mathbb{M} .
$$

The magnetic field $B$ is defined as a distribution on $\mathbb{R}^{2}$ by

$$
B:=D_{1} A_{2}-D_{2} A_{1},
$$


where $D_{j}$ is the distributional partial differential operator in $x_{j}$. Each component $A_{j}(j=1,2)$ is infinitely differentiable on $\mathbb{M}$. By the Cauchy-Riemann equation for $f$, we have

$$
B(\mathbf{x})=0, \quad \frac{\partial A_{1}(\mathbf{x})}{\partial x_{1}}+\frac{\partial A_{2}(\mathbf{x})}{\partial x_{2}}=0, \quad \mathbf{x} \in \mathbb{M} .
$$

Hence the magnetic field $B$ is concentrated, as a distribution, on the set $\mathbb{L}_{\mathbf{w}_{1}, \mathbf{w}_{2}}$. Let

$$
F_{j}^{\mathbf{A}}(\mathbf{x}):=e^{\alpha I_{\mathbf{A}}^{(j)}(\mathbf{x})}, \quad \mathbf{x} \in \mathbb{R}^{2} \backslash \mathbb{L}_{j}
$$

with $\alpha \in \mathbb{C} \backslash\{0\}$ and

$$
I_{\mathbf{A}}^{(j)}(\mathbf{x}):=\int_{\mathbf{x}}^{\mathbf{x}+\mathbf{w}_{j}} \mathbf{A}\left(\mathbf{x}^{\prime}\right) \cdot d \mathbf{x}^{\prime}, \quad \mathbf{x} \in \mathbb{R}^{2} \backslash \mathbb{L}_{j},
$$

where the integral $\int_{\mathbf{x}}^{\mathbf{x}+\mathbf{w}_{j}}$ means the line integral along the straightline $: t \mapsto$ $\mathbf{x}+t \mathbf{w}_{j}, t \in[0,1]$.

Let

$$
\Omega_{m, n}:=m w_{1}+n w_{2} .
$$

and $\operatorname{Res}\left(\Omega_{m, n}, f\right)$ be the residue of $f$ at $z=\Omega_{m, n}$.

In what follows, we assume that

$$
c_{f}:=\Re\left(\operatorname{Res}\left(\Omega_{m, n}, f\right)\right),
$$

the real part of the residue $\operatorname{Res}\left(\Omega_{m, n}, f\right)$, is a non-zero constant independently of $(m, n) \in \mathbb{Z}^{2}$. A class of such meromorphic functions $f$ is easily constructed $[1,3]$.

We introduce a constant:

$$
q_{\alpha}:=e^{2 \pi \alpha c_{f}}
$$

Lemma 2 For all $\mathrm{x} \in \Omega$,

$$
F_{1}^{\mathbf{A}}(\mathbf{x}) F_{2}^{\mathbf{A}}\left(\mathbf{x}+\mathbf{w}_{1}\right)=q_{\alpha} F_{1}^{\mathbf{A}}\left(\mathbf{x}+\mathbf{w}_{2}\right) F_{2}^{\mathbf{A}}(\mathbf{x}) .
$$

Proof. It is sufficient to show that, for each $\mathrm{x} \in \Omega$,

$$
\left.I_{\mathbf{A}}^{(1)}(\mathbf{x})+I_{\mathbf{A}}^{(2)}\left(\mathbf{x}+\mathbf{w}_{1}\right)-I_{\mathbf{A}}^{(1)}\left(\mathbf{x}+\mathbf{w}_{2}\right)-I_{\mathbf{A}}^{(2)}(\mathbf{x})\right)=2 \pi c_{f} .
$$

Let $L$ be the left hand side of this equation. It is easy to see that $L=$ $\Im \int_{C} f(z) d z$, where $C$ is the closed curve starting and ending at $x$ which is a composition of four straight lines going as $x \rightarrow x+w_{1} \rightarrow x+w_{1}+w_{2} \rightarrow$ $x+w_{2} \rightarrow x$, forming a parallelogram. There exists a unique $(m, n) \in \mathbb{Z}^{2}$ such that $\Omega_{m, n}$ is in the interior of $C$. Hence, by the residue theorem, we have $\int_{C} f(z) d z=2 \pi i \operatorname{Res}\left(\Omega_{m, n}, f\right)$. Thus $L=2 \pi c_{f}$.

We define

$$
T_{j}^{\mathbf{A}}:=F_{j}^{\mathbf{A}} V\left(\mathbf{w}_{j}\right), \quad j=1,2 .
$$


Theorem 5 Each $T_{j}^{\mathbf{A}}$ leaves $C_{0}^{\infty}(\Omega)$ invariant and

$$
T_{1}^{\mathbf{A}} T_{2}^{\mathbf{A}}=q_{\alpha} T_{2}^{\mathbf{A}} T_{1}^{\mathbf{A}}
$$

holds on $C_{0}^{\infty}(\Omega)$.

Proof. It is easy to see that the function $F_{j}^{\mathbf{A}}$ is infinitely differentiable on $\Omega$. Hence we can apply Proposition 5 with $F_{j}=F_{j}^{\mathbf{A}}$ to obtain the desired result.

Thus $\left\{T_{1}^{\mathbf{A}}, T_{2}^{\mathbf{A}}\right\}$ gives a representation of the quantum plane $\mathbb{C}_{q_{\alpha}}^{2}$ on $C_{0}^{\infty}(\Omega)$. We next consider the boundedness of this representation.

Theorem 6 Let $\Re \alpha=0$. Then each $T_{j}^{\mathbf{A}}$ is unitary.

Proof. If $\Re \alpha=0$, then $\left|F_{j}^{\mathbf{A}}\right|=1$. Hence, by Proposition 1-(ii) $T_{j}^{\mathbf{A}}$ is unitary.

In the case where $\Re \alpha \neq 0$, for each $j=1,2$, we introduce two real-valued functions $\mathbb{R}^{2} \backslash \mathbb{L}_{j}$ as follows:

$$
K_{j}(\mathbf{x}):=(\Re \alpha) \Im \int_{x}^{x+w_{j}} f(z) d z, \quad \mathbf{x} \in \mathbb{R}^{2} \backslash \mathbb{L}_{j} .
$$

Proposition 8 For each $j=1,2, T_{j}^{\mathbf{A}}$ is bounded if and only if the function $K_{j}$ is bounded above on $\mathbb{R}^{2} \backslash \mathbb{L}_{j}$.

Proof. By Proposition $1, T_{1}^{\mathbf{A}}$ and $T_{2}^{\mathbf{A}}$ are bounded if and only if $F_{1}^{\mathbf{A}}$ and $F_{2}^{\mathbf{A}}$ are essentially bounded on $\mathbb{R}^{2}$. Note that

$$
I_{\mathbf{A}}^{(j)}(\mathbf{x})=\Im \int_{x}^{x+w_{j}} f(z) d z, \quad \mathbf{x} \in \mathbb{R}^{2} \backslash \mathbb{L}_{j} .
$$

Hence $\left|F_{j}^{\mathbf{A}}(\mathbf{x})\right|=e^{K_{j}(\mathbf{x})}, \mathbf{x} \in \mathbb{R}^{2} \backslash \mathbb{L}_{j}$. Obviously each $K_{j}$ is continuous on $\Omega$. Therefore $F_{j}^{\mathbf{A}}$ is essentially bounded on $\mathbb{R}^{2}$ if and only if $K_{j}$ is bounded above on $\mathbb{R}^{2} \backslash \mathbb{L}_{j}$.

We can apply Theorem 2 to the representation $\left\{T_{1}^{\mathbf{A}}, T_{2}^{\mathbf{A}}\right\}$ of the quantum plane $\mathbb{C}_{q_{\alpha}}^{2}$ to obtain the following result:

Corollary 3 Let $a, b, a^{\prime}, b^{\prime} \in \mathbb{C}$ be constants satisfying

$$
a b q_{\alpha}=\frac{a^{\prime} b^{\prime}}{q_{\alpha}}=-\frac{1}{\left(q_{\alpha}-q_{\alpha}^{-1}\right)^{2}} .
$$

and

$$
\begin{aligned}
& E_{\mathbf{A}}:=T_{2}^{\mathbf{A}}\left(a T_{2}^{\mathbf{A}}+a^{\prime}\left(T_{2}^{\mathbf{A}}\right)^{-1}\right)\left(T_{1}^{\mathbf{A}}\right)^{-1}, \\
& F_{\mathbf{A}}:=T_{1}^{\mathbf{A}}\left(b T_{2}^{\mathbf{A}}+b^{\prime}\left(T_{2}^{\mathbf{A}}\right)^{-1}\right)\left(T_{2}^{\mathbf{A}}\right)^{-1}, \\
& K_{\mathbf{A}}:=\left(T_{2}^{\mathbf{A}}\right)^{2}, \quad K_{\mathbf{A}}^{-1}:=\left(T_{2}^{\mathbf{A}}\right)^{-2} .
\end{aligned}
$$

Then the set $\left\{E_{\mathbf{A}}, F_{\mathbf{A}}, K_{\mathbf{A}}, K_{\mathbf{A}}^{-1}\right\}$ generates a representation of $U_{q_{\alpha}}\left(\mathrm{sl}_{2}\right)$ on the vector space $C_{0}^{\infty}(\Omega)$. 


\section{Acknowledgment}

Part of this work was done during the author's stay at the Erwin Schrödinger International Institute for Mathematical Physics (ESI) in June, 2007. He would like to thank the ESI for their support.

\section{References}

[1] A. Arai: J. Math. Phys. 37, 4203-4218 (1996).

[2] A. Arai: J. Math. Phys. 39, 2476-2498 (1998).

[3] A. Arai: Representations of the quantum plane and the quantum algebra $U_{q}\left(\mathrm{sl}_{2}\right)$ on $L^{2}\left(\mathbb{R}^{d}\right)$, ESI Preprint 1942, mp_arc 07-198. This paper will not be published.

[4] C. Kassel: Quantum Groups, Springer, New York 1995.

[5] A. Klimyk and K. Schmüdgen: Quantum Groups and Their Representations, Springer, Berlin, Heidelberg 1997.

[6] K. Schmüdgen: Publ. Res. Inst. Math. Sci. 28, 1029-1061 (1992).

[7] K. Schmüdgen: Commun. Math. Phys. 159, 217-237 (1994).

[8] K. Schmüdgen: Lett. Math. Phys. 37, 211-222(1996).

[9] P. B. Wiegmann and A. V. Zabrodin: Nuclear Phys. B 422, 495-514 (1994). 